\title{
LOWER BOUND OF ZERO-VISIBILITY COPS AND RobBer GaMe ON THE CUBE GRID
}

\author{
Jiahui Wang and Farong Zhong \\ Department of Computer Science, Zhejiang Normal University, Jinhua, China.
}

\begin{abstract}
Zero-visibility cops and robber game is a variant of the classical model. The robber is invisible in the zero-visibility game. In this paper, we study the zero-visibility cops and robber game on the cube grid. We first study a partition problem of the cube grid $C_{n^{3}}$. Then we prove the lower bound on the zero-visibility cop number of the cube grid by using the results in the partition. We also show the lower bound is a quadratic polynomial about $n$.
\end{abstract}

\section{KEYWORDS}

Cube Grid; Cops and robber; Partition; Zero-visibility Cop number

\section{INTRODUCTION}

Cops and robber game is an interesting pursuit-evasion game in graph theory, which was introduced by Nowakowski and Winkler [1] and Quilliot [2] in 1983. We consider the game of cops and robber on a graph $G$. There are two kinds of players: one robber and multiple cops. In each round, the cops move first and then the robber. The player can only move to the adjacent vertex or stay still. The robber is captured if a cop and the robber occupy the same vertex in a finite number of moves. Our goal is to determine the minimum number of cops to capture the robber.

Cops and robber game can be divided into three models based on visibility. In these three models, the robber knows the location of all cops at any time, but the cop's visibility of the robber is different. The first is that the cops know the perfect information of the robber, a lot of results can be found in $[3,4,5,6]$. The second is that the cops know the imperfect information of the robber. The visibility of the cops is limited to the distance between the cops and the robber within $\mathrm{k}$ range, so this model is called the k-visibility cops and robber game. In this case, related results can be found in $[7,8]$.

Zero-visibility cops and robber game is the third model, in which the cops have no information about the location of the robber, the robber is invisible. The zero-visibility cop number of $G$, denoted $c_{0}(G)$, is the minimum number of cops needed to capture the invisible robber, and a strategy used $c_{0}(G)$ cops is called the optimal search strategy. It was first proposed by Tošić [9] in 1985. Zero-visibility model makes the problem more difficult, so there are not many results. The key to determine $c_{0}(G)$ is to find the lower bound. Yang [10] proposed a partition method, which can be applied to prove the lower bound of $c_{0}(G)$. this method only used for simple 
cartesian products in [10]. Cube grid is a three-dimensional graph, so we use this method on the cube grid. Given an integer $k$, and find a partition of the cube grid such that the size of the

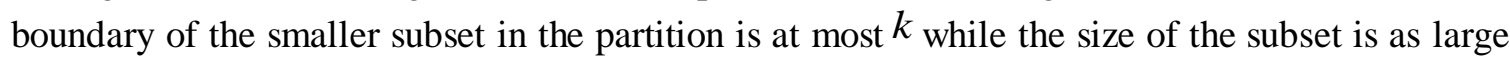
as possible [10]. This method can successfully help us determine the lower bound of $c_{0}\left(G_{n^{3}}\right)$,

The rest of this paper is as follows. In section 2, we give the related work. In section 3, we give some definitions. In section 4 , we study a partition problem of the cube grid. In section 5, we show the lower bound of $c_{0}\left(C_{n^{3}}\right)$ is a quadratic polynomial about $n$, and the last section is a summary.

\section{RELATED WORK}

Tošić [9] studied the zero-visibility cop numbers of paths, cycles, complete graphs, and complete bipartite graphs. Tang [11] proposed a quadratic time algorithm to determine the zero-visibility cop number of a tree, then a linear algorithm of a tree was given by Dereniowskl [12]. In the above articles, only simple graphs were studied, and there is no better method to determine the zero-visibility cop number until Dereniowsk1 [13] studied the relationship between the monotonic zero-visibility cop number and the path width of a graph. Some scholars have calculated the path width of different graphs in [14], also the path width of the cube grid can be found in [15]. But how to determine the lower bound of $c_{0}(G)$ is also difficult. Yang [10] proposed to use the partition method to prove the lower bound of the cop number in a simple Cartesian product graph, and new results of this method to determine the zero-visibility cop number can be found in [16].

\section{Preliminaries}

Consider the zero-visibility cops and robber game on a graph $G(V, E)$ with vertex $\operatorname{set} V$ and edge set $E$, which can be seen as a node-search problem. First, make all vertices contaminated, then the robber can only be on contaminated vertices. Thus, the search is successful if the givencop number and search strategy can make all vertices clean.

Let $G_{\text {and }} G^{\prime}$ be two graphs, the cartesian product of $G_{\text {and }} G^{\prime}$, denoted by $G \square G^{\prime}$, is the graph in which $V\left(G_{\square} G^{\prime}\right)=V(G) \times V\left(G^{\prime}\right)$, and two vertices $(u, v)$ and $\left(u^{\prime}, v^{\prime}\right)$ are adjacent in $G \square G^{\prime}$ if and only if $u=u^{\prime}, v_{\text {and }} v^{\prime}$ are adjacent in $G^{\prime}$; or $v=v^{\prime}, u$ and $u^{\prime}$ are adjacent in $G$. Let ${ }_{n}$ be a path

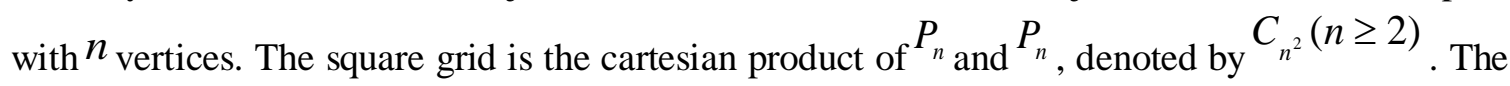
cube grid is the cartesian product of $C_{n^{2}}$ and $P_{n}$, denoted by $C_{n^{3}}(n \geq 2)$, which has $n^{3}$ vertices. we define the direction in the cubic grid, let $d:\{x, y, z\}$, see in Figure 1. In each direction, there are $n_{\text {copies of }} C_{n^{2}}$ and $n^{2}$ copies of $P_{n} C_{3^{2}}$ and $C_{3^{3}}$ shown in Figure 1.

Let $\left(V_{1}, V_{2}\right)$ be a partition of $G(V, E)$ if $V_{1} \cup V_{2}=V$ and $V_{1} \cap V_{2}=\phi$. Two vertices ${ }^{v_{i}}$ and ${ }^{v_{j}}$ are adjacent, denoted by $v_{i} \sim v_{j}$. Let $V_{\mathrm{B}}$ be the boundary of $V_{1}$ if $V_{\mathrm{B}} \subseteq V_{1}$,

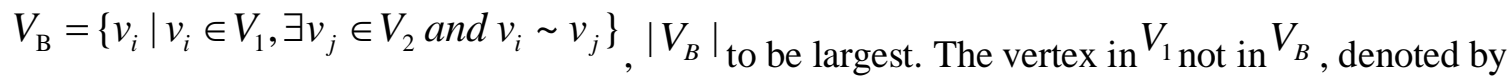


$V_{C}$, we have $V_{C}=V_{1}-V_{B}$. In the zero-visibility cops and robber searching model, the contaminated and cleared vertices always changed after each round. Let $V_{B}^{i}(i \geq 1)$ be the boundary after the cops' move in round $i, V_{1}^{i}, V_{2}^{i}$ be the set of the cleared and contaminated vertices after the robber' move in round ${ }^{i} .\left(V_{1}^{i}, V_{2}^{i}\right)$ is a partition of $G$ after the robber' move in round $i$.

Each direction contains all vertices of the cube grid, so we take the $\mathrm{z}$ direction as an example. Let $C_{n^{2}}^{l}(1 \leq l \leq n)$ be the $l$-th $C_{n^{2}}$ in the z direction, $v_{l, t}\left(1 \leq t \leq n^{2}\right)$ be the $t$ - th vertex in the $C_{n^{2}}^{l}$ (see in figure 1). Let $r_{l}, p_{l}, q_{l}$ be the number of vertices in $V_{1}, V_{C}, V_{B}$ of $C_{n^{2}}^{l}(1 \leq l \leq n)$ respectively, we have $r_{l}=p_{l}+q_{l}$.
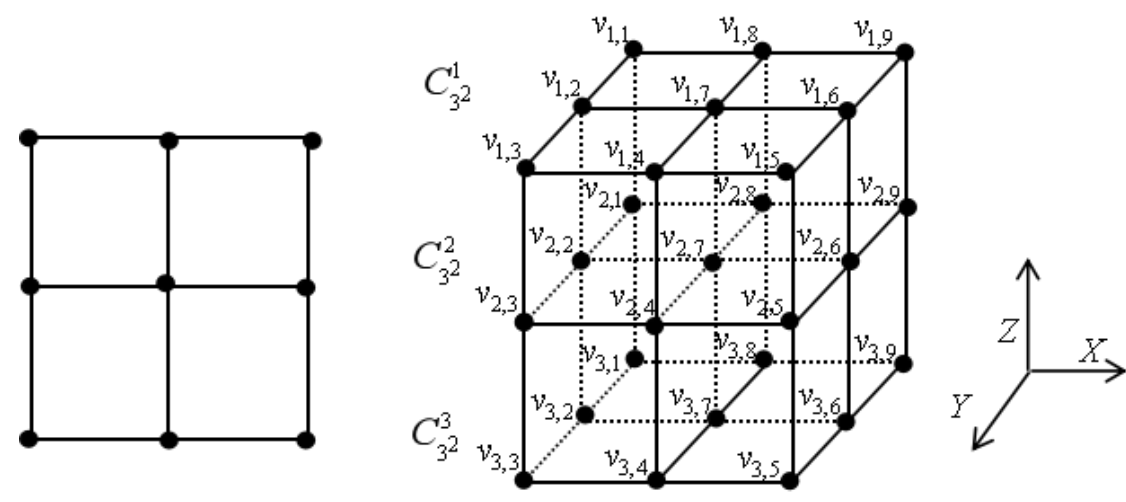

Figure 1. Square grid $C_{3^{2}}$, Cube grid $C_{3^{3}}$.

\section{Partition of the Cube Grid}

Lemma 1. Let $\left(V_{1}, V_{2}\right)$ be a partition of $C_{n^{2}}$ for $n \geq 2$. If $\left|V_{1}\right|=\frac{m(m+1)}{2}$ and $1 \leq m \leq n$, then $\left|V_{B}\right| \geq m$.

Proof. The claim is true when $m=1$. For the sake of contradiction, suppose that $\left|V_{B}\right|<m_{\text {when }}$ $2 \leq m \leq n$. If there exist $m$ rows or $m$ columns that contain both vertices in $V_{1}$ and $V_{2}$, then $\left|V_{B}\right| \geq m$, which is a contradiction. Hence, there are at least $n-m+1$ rows and $n-m+1$ columns that contain vertices only in $V_{1}$ or $V_{2}$. Here we have two cases.

Case 1: There exist $n-m+1$ rows and $n-m+1$ columns that contain vertices only in $V_{2}$. In this case, it can be seen that there exist a row and a column contain vertices only in $V_{2}$ of $C_{m^{2}}$. From theorem 1 in Yang [15], we have $\left|V_{1}\right| \leq \frac{m(m-1)}{2}<\frac{m(m+1)}{2}$. 
Case2: There exist $n-m+1$ rows and $n-m+1$ columns that contain vertices only in $V_{1}$. Note that $\left|\mathrm{V}_{1}\right|_{\min }=n^{2}-\left|V_{2}\right|_{\max }$, the maximum of $\left|V_{2}\right|_{\text {is equal to the maximum of }}\left|V_{C}\right|_{\text {in case } 1 \text {,so }}$ consider case 1 , we will show that when $\left|V_{C}\right|_{\max }=w,\left|V_{B}\right|=m-1$. Assume that $\left|V_{B}\right|=k<m-1$, $\left|V_{C}\right|_{\max }=w$. If there are $m-1$ rows or $m-1$ columns contain both vertices in $V_{1}$ and in $V_{2}$, then $\left|V_{B}\right| \geq m-1$, which is a contradiction. Thus, there are at least $n-m+2$ rows and $n-m+2$ columns that contain vertices only in $V_{2}$. Let ${ }^{{ }^{i}}$ be a vertex in $V_{2}$, satisfied $\left\{v_{i} \mid v_{i} \sim v_{j}, v_{j} \in V_{B}\right\}$, then let $v_{i} \in V_{1}$, we can obtain a new partition of $C_{n^{2}}$. In a new partition, we have $\left|V_{B}\right|=k$, $\left|V_{C}\right|=w+1$; or $\left|V_{B}\right|=k+1,\left|V_{C}\right|=w$, which is a contradiction. Thus, when $\left|V_{C}\right|_{\max }=w$, we obtain $\left|V_{B}\right|=m-1$. Since $w=\left|V_{1}\right|-(m-1),\left|V_{1}\right| \leq \frac{m(m-1)}{2}$, we have $w \leq \frac{m(m-1)}{2}-(m-1)$. Hence, in case $2, \quad\left|\mathrm{~V}_{1}\right|_{\min }=n^{2}-\left[\frac{m(m-1)}{2}-(m-1)\right]$. Therefore, $\left|V_{1}\right| \geq \frac{m(m+3)}{2}-1>\frac{m(m+1)}{2}(m \geq 2)$

See in figure 2.

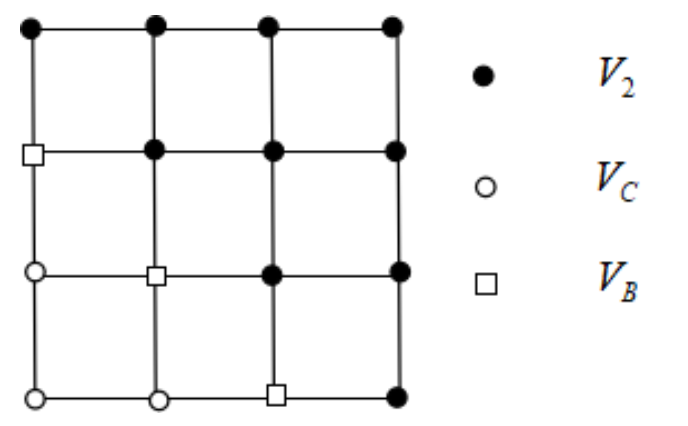

Figure 2. $C_{4^{2}}$ with $m=3,\left|V_{1}\right|=6,\left|V_{B}\right|=3$.

Lemma 2. Let $\left(V_{1}, V_{2}\right)$ be a partition of $C_{n^{3}}$ for $n \geq 2$. If $\max \left\{r_{l} \mid 1 \leq l \leq n\right\}=m\left(m \leq \frac{n(n+1)}{2}\right)$, then $\left|V_{B}\right| \geq m$.

Proof. We know that on a $C_{n^{3}}(n \geq 2)$, if $r_{l_{1}} \leq r_{l_{2}} \leq \frac{n(n+1)}{2},\left(1 \leq l_{1}, l_{2} \leq n\right)$, then $q_{l_{1}} \leq q_{l_{2}}$. So $\left|V_{B}\right|=\sum_{l=1}^{n} q_{l}$ is the minimum when $r_{1}=m$ or $r_{n}=m$, this two cases are similar. Thus, take $r_{n}=m$ as an example, there will be $r_{l-1} \leq r_{l}, q_{l-1} \leq q_{l}(2 \leq l \leq n)$. Here are two cases. 
Case 1: $\min \left\{r_{l} \mid 1 \leq l<n\right\}=0$.Suppose that ${ }^{r_{i}}=0$, then let ${ }^{v_{m, t}}$ be a vertex in $C_{n^{2}}^{m}$ such that $v_{m, t} \in V_{1}$, there exist a path $v_{i, t} \ldots v_{m, t}\left(v_{i, t} \in C_{n^{2}}^{i}, v_{i, t} \in V_{2}\right)$ in z direction, and this path must contain a vertex in $V_{B}$. Since $r_{i}=m$, we have $\left|V_{B}\right| \geq m$. (see in figure 3)

Case 2: $\min \left\{r_{l} \mid 1 \leq l<n\right\}>0$.Suppose that $r_{n}=\frac{n(n+1)}{2}$, then $q_{n} \geq n$ from lemma 1. And let $q_{n}=n$ for minimum, we obtain $1 \leq q_{l} \leq n$, and we have $q_{l-1} \leq q_{l}$ when $\left|V_{B}\right|_{\text {is the minimum, so }}$ there exists one copy of $C_{n^{2}}$ that contain one vertex in $V_{B}$. Since $r_{n}=m<\frac{n(n+1)}{2}$, there are at least two copies of $C_{n^{2}}$ in which the number of boundaries equals 1 when $\left|V_{B}\right|_{\text {is the minimum. }}$ Let $C_{n^{2}}^{j}$ be a copy of $C_{n^{2}}$ in which $r_{j}=q_{j}=1$. Suppose that there is no copy of $C_{n^{2}}$ between $C_{n^{2}}^{j}$ and $C_{n^{2}}^{n}$ in which the boundary number equals 1 , similar to (1), we have $\left|V_{B}\right| \geq m$. And there exist another copy of $C_{n^{2}}$ whose boundary number equals 1 , so we have $\left|V_{B}\right| \geq m+1$.(see in figure 4)

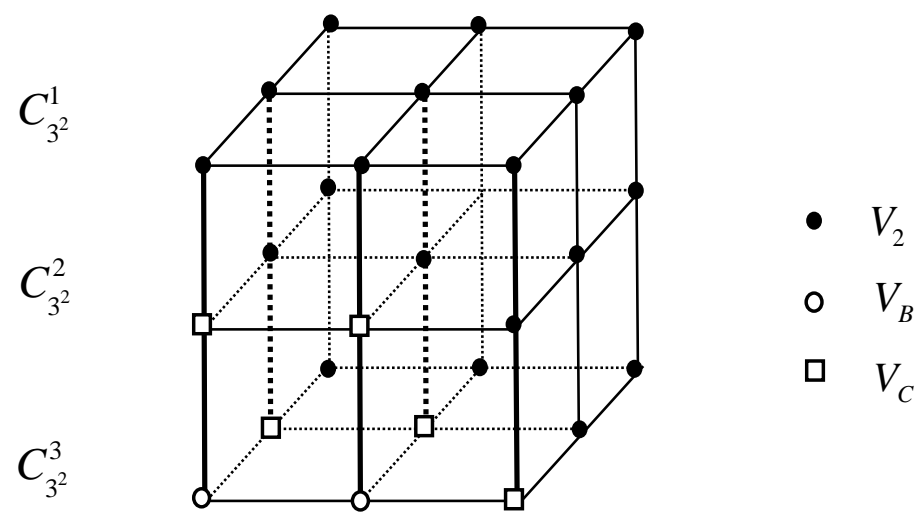

Figure 3. $C_{3^{3}}$ with $i=1, r_{i}=0, m=5$, and there exist 5 copies of $P_{3}$ (bold lines)which contain a vertex in $V_{1}$ and a vertex in $V_{2}$, so $\left|V_{B}\right|=5$.

Theorem 1. Let $\left(V_{1}, V_{2}\right)$ be a partition of $C_{n^{3}}$ for $n \geq 2$. If $\left|V_{B}\right|<\frac{n(n+1)}{2}+1$, and there exist $\frac{n(n-1)}{2}$ copies of $P_{n}$ in which all vertices belong to $V_{2}$ in one direction, then $\left|V_{1}\right| \leq \sum_{l=1}^{n} \frac{l(l+1)}{2}$.

Proof. Take z direction as an example. Since there exist $\frac{n(n-1)}{2}$ copies of ${ }^{P_{n}}$ whose vertices all belong to $V_{2}$, then $r_{l} \leq \frac{n(n+1)}{2}(1 \leq l \leq n)$. We need to find the maximum of $\left|V_{1}\right|$. Suppose that $\max \left\{r_{l}(1 \leq l \leq n)\right\}=\frac{n(n+1)}{2}$, from lemma2, we have $\left|V_{B}\right| \geq \frac{n(n+1)}{2}$.And since 
$\left|V_{B}\right|<\frac{n(n+1)}{2}+1$, we have $\left|V_{B}\right|=\frac{n(n+1)}{2}$. If $C_{n^{2}}^{i}, C_{n^{2}}^{i+\ldots . . . C_{n^{2}}^{j}(1 \leq i \leq j \leq n) \text { contain vertices }}$ in $_{1} V_{1}$ then $r_{k-1}=p_{k-1}+q_{k-1} \geq p_{k}(i+1 \leq k \leq j)$. Hence, when $\left|V_{B}\right|=\frac{n(n+1)}{2}$, there will be $r_{k-1}=p_{k}(i+1 \leq k \leq n), p_{i}=0, r_{i}=q_{i}, r_{n}=\frac{n(n+1)}{2}$. And from lemma1 $q_{n} \geq n$. Since $r_{k-1}=p_{k}$, we have $r_{n-1}=p_{n} \leq \frac{n(n+1)}{2}-n=\frac{n(n-1)}{2}$.Let $r_{n-1}=\frac{n(n-1)}{2}$ for maximum, also from lemma 1, there will be $q_{n-1} \geq n-1$, and so on. Thus, when $\left|V_{1}\right|_{\text {is the maximum, we have }} r_{l}=\frac{l(l+1)}{2}$, $q_{l}=l(1 \leq l \leq n)$, satisfied $\left|V_{B}\right|=\sum_{l=1}^{n} q_{l}=\frac{n(n+1)}{2}<\frac{n(n+1)}{2}+1$. Therefore, $\left|V_{1}\right| \leq \sum_{l=1}^{n} \frac{l(l+1)}{2}$.(see in figure 4)

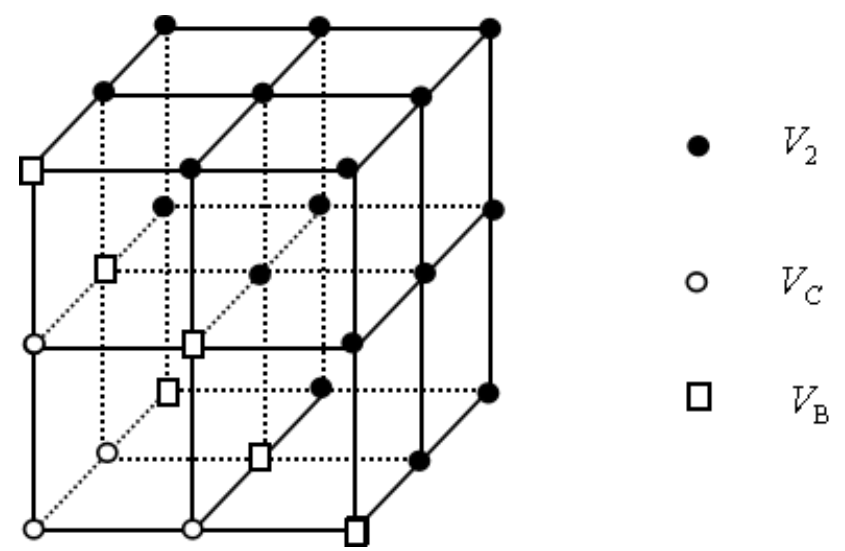

Figure 4. $C_{3^{3}}$ with $\left|V_{B}\right|=6,\left|V_{1}\right|=10$.

\section{LOWER BOUND OF ZERO-VISIBILITY COP NUMBER}

Lemma 3([15]). Let $G$ be a graph with ${ }^{c_{0}(G)=k}$. For any round ${ }^{i(i \geq 1)}$ of an optimal search strategy of $G$, if $\left|V_{B}^{i}\right| \geq 2 k$, then $\left|V_{1}^{i}\right| \leq\left|V_{1}^{i-1}\right|$.

Lemma4. For an optimal search strategy of $C_{n^{3}}$, if $\left|V_{B}^{i}\right|<\frac{n(n+1)}{2}+1$, then there will be

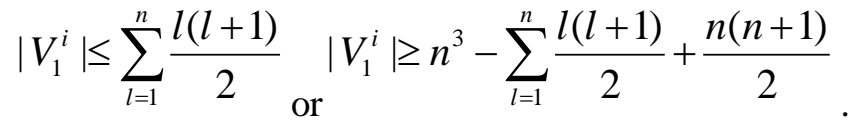


Proof. After cops' move in round i, take z direction as an example, there are $n^{2}$ copies of $P_{n}$ in z direction. If there are $\frac{n(n+1)}{2}+1$ copies of $P_{n}$ that contain both cleared vertices and contaminated vertices, then $\left|V_{B}^{i}\right| \geq \frac{n(n+1)}{2}+1$, which is a contradiction. Hence, there are at least $\frac{n(n-1)}{2}$ copies of $P_{n}$ in which all vertices are cleared or contaminated. Here we have two cases.

Case1: There exist $\frac{n(n-1)}{2}$ copies of $P_{n}$ that contain only contaminated vertices. Let $V_{1}, V_{2}$ be the set of cleared vertices and contaminated vertices respectively after cops' move in round $i$. From theorem1, we have $\left|V_{1}\right| \leq \sum_{l=1}^{n} \frac{l(l+1)}{2}$. Therefore, $\left|V_{1}^{i}\right| \leq\left|V_{1}\right| \leq \sum_{l=1}^{n} \frac{l(l+1)}{2}$. Case2: There exist $\frac{n(n-1)}{2}$ copies of $P_{n}$ that contain only cleared vertices. The proof is similar to lemma1, consider case 1 again, we will show that when $\left|V_{B}^{i}\right|=\frac{n(n+1)}{2},\left|V_{1}\right|-\left|V_{B}^{i}\right|$ is the maximum. Thus, in case2, the maximum number of contaminated vertices is $\sum_{l=1}^{n} \frac{l(l+1)}{2}-\frac{n(n+1)}{2}$. Therefore, we have $\left|V_{1}^{i}\right| \geq n^{3}-\sum_{l=1}^{n} \frac{l(l+1)}{2}+\frac{n(n+1)}{2}$.

Theorem 2.For $n \geq 2, c_{0}\left(C_{n^{3}}\right) \geq\left\lfloor\frac{n(n+1)}{4}+\frac{3}{2}\right\rfloor$.

Proof. For the sake of contradiction, suppose that $c_{0}\left(C_{n^{3}}\right) \leq\left\lfloor\frac{n(n+1)}{4}+\frac{1}{2}\right\rfloor$, and consider a strategy that uses at most $\left\lfloor\frac{n(n+1)}{4}+\frac{1}{2}\right\rfloor_{\text {cops, we will show that }}\left|V_{1}^{i}\right| \leq \sum_{i=1}^{n} \frac{l(l+1)}{2}<n^{3},(i \geq 0)$. That means $\left\lfloor\frac{n(n+1)}{4}+\frac{1}{2}\right\rfloor$ cops can't make all vertices clean, which is a contradiction. When $i=0$, we have $\left|V_{1}^{0}\right| \leq\left\lfloor\frac{n(n+1)}{4}+\frac{1}{2}\right\rfloor \leq \sum_{l=1}^{n} \frac{l(l+1)}{2} \quad$; then assume that $\left|V_{1}^{i-1}\right| \leq \sum_{l=1}^{n} \frac{l(l+1)}{2}$, here we have two cases to prove $\left|V_{1}^{i}\right| \leq \sum_{l=1}^{n} \frac{l(l+1)}{2}$.

Case1: $\left|V_{B}^{i}\right| \geq \frac{n(n+1)}{2}+1$. From lemma 3, $\left|V_{1}^{i}\right| \leq\left|V_{1}^{i-1}\right|$. Therefore, $\left|V_{1}^{i}\right| \leq \sum_{l=1}^{n} \frac{l(l+1)}{2}$. 
Case2: $\left|V_{B}^{i}\right|<\frac{n(n+1)}{2}+1$. Since $\left|V_{1}^{i-1}\right| \leq \sum_{l=1}^{n} \frac{l(l+1)}{2}$, there are at most $\left\lfloor\frac{n(n+1)}{4}+\frac{1}{2}\right\rfloor+\sum_{l=1}^{n} \frac{l(l+1)}{2}$ cleared vertices after cops' move in round $i$. Also, since $n^{3}-\sum_{l=1}^{n} \frac{l(l+1)}{2}+\frac{n(n+1)}{2}>\left\lfloor\frac{n(n+1)}{4}+\frac{1}{2}\right\rfloor+\sum_{l=1}^{n} \frac{l(l+1)}{2}, \quad$ we $\quad$ have $_{1}^{i} \mid \leq \sum_{l=1}^{n} \frac{l(l+1)}{2}$ from lemma 4.

\section{Conclusion}

In this paper, we successfully apply the partition method to study zero-visibility cops and robber game on the cube grid and show the lower bound of $c_{0}\left(C_{n^{3}}\right)$. Perhaps the lower bound obtained by this method is not the best, But we narrow the scope of finding the final solution. In the future, we will use the result in this paper to find the exact value of $c_{0}\left(C_{n^{3}}\right)$ and also use this method on other three-dimensional graph.

\section{REFERENCES}

[1] R. Nowakowski and P. Winkler(1983). Vertex to vertex pursuit in a graph. Discrete Mathematics 43:235-239.

[2] Quilliot, A.(1983): Problèmes de jeux, de point fifixe, de connectivité et de représentation sur des graphes, des ensembles ordonnés et des hypergraphes. $\mathrm{PhD}$ thesis, Université de Paris VI

[3] Anthony Bonato. (2011). The game of cops and robbers on graphs. Student Mathematical Library, 276.

[4] Anthony Bonato, \& Boting Yang. (2013). Graph searching and related problems. Handbook of Combinatorial Optimization, 1511-1558.

[5] Bonato, A. , \& Macgillivray, G. . (2017). Characterizations and algorithms for generalized cops and robbers games.

[6] Brandt, S. , Emek, Y., Uitto, J. , \& Wattenhofer, R. . (2020). A tight lower bound for the capture time of the cops and robbers game. Theoretical Computer ence.

[7] Isler, V., Karnad, N.(2008): The role of information in the cop-robber game. Theoretical Computer Science 399, 179-190

[8] Clarke, N. E. , Cox, D. , Duffy, C. , Dyer, D. , \& Messinger, M. E. . (2019). Limited visibility cops and robber. Discrete Applied Mathematics, 282.

[9] Tošić R.(1985), Vertex-to-Vertex Search in a Graph, Proceedings of the Sixth Yugoslav Seminar on Graph Theory, 233-237.

[10] Xue, Y. , Yang, B. , Zhong, F. , \& Zilles, S. . (2019). A Partition Approach to Lower Bounds for Zero-Visibility Cops and Robber. Combinatorial Algorithms.

[11] Tang, A.(2004): Cops and robber with bounded visibility[D]. Master's thesis, Dalhousie University.

[12] Dereniowski, D. , Dyer, D. , Tifenbach, R. M. , \& Yang, B. . (2014). The complexity of zerovisibility cops and robber.

[13] Dariusz Dereniowski, Danny Dyer, Ryan M. Tifenbach, \& Boting Yang. (2013). Zero-visibility cops and robber game on a graph. Lecture Notes in Computer Science, 7924, 175-186.

[14] Ellis, J. , \& Warren, R. . (2008). Lower bounds on the pathwidth of some grid-like graphs. Discrete Applied Mathematics.

[15] Otachi, Y. , \& Suda, R. . (2011). Bandwidth and pathwidth of three-dimensional grids. Discrete Mathematics, 311(10), 881-887.

[16] Xue, Y. , Yang, B. , \& Zilles, S. . (2019). New Results on the Zero-Visibility Cops and Robber Game. International Conference on Algorithmic Applications in Management. Springer, Cham. 


\section{AUTHORS}

Jiahui Wang: an M.S. candidate in Computer Science at Zhejiang Normal University. Her research interests focuses on Graph searching.

Farong Zhong: a professor in Computer Science at Zhejiang Normal University. He received his B.S.degree in Computer Science from Shandong University in 1986, M.S. and Ph.D. degree in Computer Science from Shanghai Jiaotong University in 1994 and 2005 respectively. His research interests focuses on process calculus, Web services, and Graph searching.

(C) 2020 By AIRCC Publishing Corporation. This article is published under the Creative Commons Attribution (CC BY) license. 